\title{
correction
}

\section{A histone fold TAF octamer within the yeast TFIID transcriptional coactivator}

William Selleck, Ryan Howley, Qiaojun Fang, Vladimir Podolny, Michael G. Fried, Stephen Buratowski and Song Tan

Nature Structural Biology 8, 695-700 (2001).

In this paper, we analyzed genetic interactions between several TAF (TBP-associated factor) subunits in yeast. The phenotype of one temperature sensitive allele of TAF48 (a H2A-like TAF) listed in Table 1 was attributed to the N204D mutation in this protein. However, due to a sequence analysis error, a second mutation (L365P) within this same allele was not recognized. Upon separation of the two mutations, it became clear that L365P confers the temperature sensitive phenotype. Although this finding does not change the conclusions of the paper, it should be noted that Leu 365 is just C-terminal to, rather than within, the canonical histone fold. We sincerely regret any inconvenience this error may have caused. 